\title{
A Spin Glass with Random Couplings
}

\author{
P. Collet, ${ }^{1}$ J.-P. Eckmann, ${ }^{2}$ V. Glaser, ${ }^{3,4}$ and A. Martin ${ }^{3}$
}

Received December 22, 1983

We define and study a spin glass model based on a RG analysis of its random couplings. The Edwards-Anderson parameter shows a transition.

KEY WORDS: Spin glasses; random coupling; Edward-Anderson transition.

\section{INTRODUCTION}

Spin-glass models still present something of a mystery: It is not exactly known what they should describe, and therefore it is not obvious what makes a spin-glass model "realistic" or "relevant." Certainly the review by Toulouse $^{(1)}$ vividly describes the ambiguous situation in the field. We therefore feel free to add, or rather reemphasize, a commonly somewhat neglected aspect of the question: Namely, we want to view the spin-glass problem as a problem of the random variables describing the random couplings. In particular, we are interested in the behavior of the effective random coupling under a change of scale. This will lead us naturally to a renormalization group ( $R G)$ approach.

This description will become exact in the hierarchical approximation described below (see also Refs. 2 and 3), and we shall describe and study some aspects of the corresponding models which are random version of a Migdal-Kadanoff type of recursion relation. ${ }^{(4,5)}$ Alternatively our approach leads us to a study of nonindependent (but not strongly coupled (mean-field $\left.{ }^{(6)}\right)$ ) random variables, and our results can be viewed as an example of nontrivial behavior in this field of mathematics.

\footnotetext{
'Ecole Polytechnique, Palaiseau, France.

${ }^{2}$ IHES, Bures/Yvette, France; on leave from the University of Geneva.

${ }^{3}$ CERN, Genève, Switzerland.

${ }^{4}$ With great sadness we have to inform the reader that Vladimir Jurko Glaser died on January $22,1984$.
} 
The purpose of our paper is to describe and analyze a class of such models, and in particular to study the "evolution" of the effective random coupling as a function of the size of the lattice (Sections 1,2, and 3).

In Section 4, we shall then arrive at the proof of existence of a spin-glass transition in the following sense. For a class of random interactions, we show that at high temperature the expected value of the spin is

$$
E(\langle s\rangle)=0
$$

and

$$
E\left(\langle s\rangle^{2}\right)=0
$$

where \langle\rangle denotes the partition sum and $E(\cdot)$ denotes averaging over the sample space of random couplings. At low temperature, we have

$$
E(\langle s\rangle)=0
$$

but

$$
E\left(\langle s\rangle^{2}\right) \neq 0
$$

indicating a transition of the Edwards-Anderson parameter. We are unable to locate a critical surface, although we can exhibit a critical fixed point.

In order to study this question, we consider a simplifed model in a separate publication ${ }^{(7)}$ in which the probability density $\rho$ of the random coupling is replaced by a discrete sequence

$$
\rho_{n} \sim \int_{1 / 2^{n+1}}^{1 / 2^{n}}(\rho(x)+\rho(-x)) d x
$$

and the renormalization transformation is replaced by the simpler, but-as we believe-qualitatively correct operator defining $\rho_{n}^{\prime}$ from $\rho_{n}$ by

$$
\rho_{n}^{\prime}=\sum_{p+q=n+1} \rho_{p} \rho_{q}+\delta_{n 0} \rho_{0}^{2}
$$

This operator, $T\left\{\rho_{n}\right\}=\left\{\rho_{n}^{\prime}\right\}$, has two fixed points

$$
\begin{array}{ll}
\left\{\rho_{n}\right\}=\{1,0,0, \ldots,\} \equiv S_{L} & \text { (low temperature) } \\
\left\{\rho_{n}\right\}=\{0,1,0, \ldots,\} \equiv S_{C} & \text { (critical temperature) }
\end{array}
$$

and a "stable" limit

$$
\left\{\rho_{n}\right\}=\{0,0, \ldots, 0,1\} \equiv S_{H} \quad \text { (i.e., } \rho_{\infty}=1 \text { ) (high temperature) }
$$

We show among other things that only three fates can happen to a sequence under iteration by $T$. If it is $S_{C}$ it is a fixed point. Otherwise the sequence tends to $S_{L}$ or to $S_{H}$, and, most interestingly, there is a critical surface $\mathscr{H}$, and every sequence on it (except $S_{C}$ ) tends to $S_{L}$. More 
precisely, define $f(s)=\sum_{n=0} \rho_{n} s^{n}$. The action of $T$ on these generating functions is $(T f)(s)=\left(f(s)^{2}-f(0)^{2}\right) / s+f(0)^{2}$. Then the critical surface is given by the equation $f(2)-2 f^{\prime}(2)=0$, while the high-temperature (h.t.) region is $f(2)-2 f^{\prime}(2)<0$, and the low-temperature region is $f(2)-2 f^{\prime}(2)$ $>0$. Note that the speed with which the critical surface is left on the h.t. side depends on the initial distribution and thus no critical indices are really defined. This may account for some of the difficulties encountered in spin glasses.

\section{THE MODELS}

The models we are considering have two equivalent formulations: One is based on a construction of Migdal-Kadanoff, ${ }^{(4,5)}$ while the other is based on the recursive diamond shaped lattices (see Ref. 2).

In the first case, we consider a $\mathbb{Z}^{d}$ lattice with an Ising spin at each site. In this lattice, we single out a direction, for example the first coordinate $\mathbf{e}=(1,0, \ldots, 0)$, and we assume that the system has side $2^{N}$ in the $\mathbf{e}$ direction and $2^{N}-1$ in the others. The following description should be easier to understand by referring to Fig. 1.

To each (horizontal) link of the form $\mathbf{i}, \mathbf{i}+\mathbf{e}, \mathbf{i} \in \mathbb{Z}^{d}$ there is a random coupling $\xi_{\mathbf{i}}$. Below, we shall specify the nature, independence, ... of the random variables $\xi_{i}$. There are now two types of interaction between the Ising spins.

(i) The interaction energy between $s_{\mathbf{i}}$ and $s_{\mathbf{i}+\mathbf{e}}$ is $\xi_{\mathbf{i}}$.

(ii) Every hyper-"plane" with fixed first coordinates $i_{1}>0$ is partitioned into $2^{(N+1-r)(d-1)}$ hypercubes of dimension $d-1$ and of side $2^{r}-1$, where $r$ is given by

$$
i_{1}=2^{r}+2^{r_{2}}+\cdots+2^{r_{k}}, \quad r<r_{2}<r_{3}<\cdots<r_{k}
$$

$i_{1}=0$ is handled as $i_{1}=2^{N}$. There is an infinite ferromagnetic coupling in each hypercube, i.e., all spins in one such hypercube are equal.

For a fixed choice of the random variables $\boldsymbol{\xi}=\left\{\xi_{\mathbf{i}}\right\}$, we denote by $H_{N}(\mathbf{s}, \xi)$ the energy of the spin configuration $\mathbf{s}$. The Gibbs density at inverse temperature $\beta$ is given by

$$
G_{n}(\mathbf{s}, \xi, \beta)=\frac{\exp \left[-\beta H_{N}(\mathbf{s}, \xi)\right]}{\sum_{\mathbf{s}^{\prime}} \exp \left[-\beta H_{N}\left(\mathbf{s}^{\prime}, \xi\right)\right]}
$$

We are interested in the properties of $G_{N}$ as a function of $\beta$ in the thermodynamic limit, $N \rightarrow \infty$.

We now give a second description of the model, using a recursive buildup of a (hierarchical) lattice. This formulation has a natural extension 

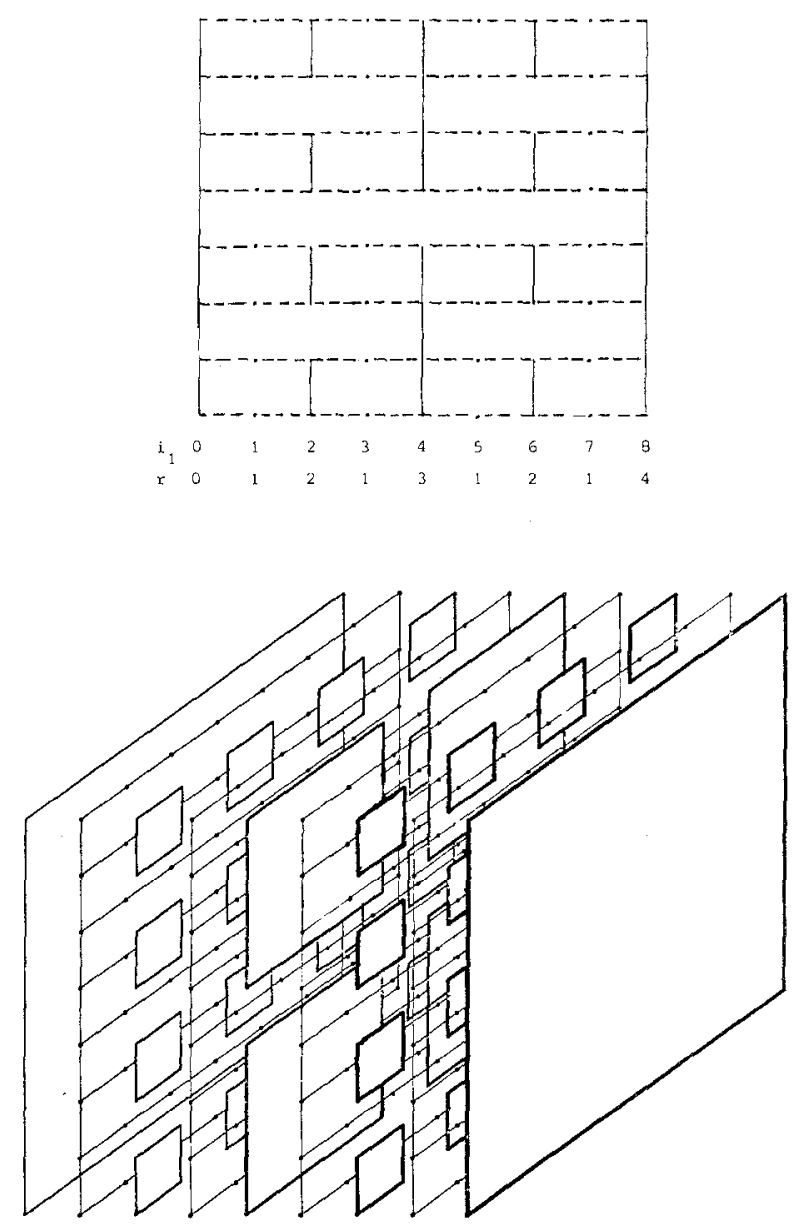

Fig. 1. (a) Lattice for $d=2, N=3$, e horizontal. (b) Lattice for $d=3, N=3$, e horizontal.

to noninteger dimensions, as we shall see. One first chooses an integer $n \geqslant 1$ (one should think of $n=2^{d-1}$, in the first formulation of the model). The lattice is then formed recursively as follows. The first lattice is formed by two sites, and one link (see Fig. 2).

We call this $L_{0}$. If $L_{p}, p \geqslant 0$, has been constructed, then $L_{p+1}$ is obtained by replacing each link by $n$ sites and $2 n$ links connecting each new site to the two ends of the original link (see Fig. 3).

We now consider $L_{N}$. To each site $i$ (numbered in some suitable fashion) we associate an Ising spin, and to each link $(i, j)$ a random coupling constant $\xi_{i, j}$. Again, for each fixed choice of the random variables, 


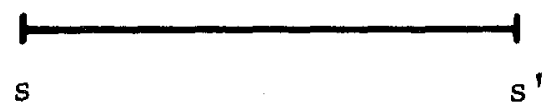

Fig. 2. One bond.

we can define

$$
H_{N}(\mathbf{s}, \xi)=\sum_{\substack{i, j \\ \text { (nearest neighbors) }}} \xi_{i, j} s_{i} s_{j}
$$

The Gibbs measure is defined as before.

So far, we have said nothing about the nature of the random variables, and the model still leaves us some freedom of choice. The most interesting choice is that of independent (identically distributed) random variables. We discuss this case in a separate publication. ${ }^{(8)}$ The main difference of that variant as contrasted to the one presented below is the presence of frustration. But it should be stressed that even in the simplified model of this paper, the absence of frustration is not the same as talking about a purely ferromagnetic interaction, as we shall see. Alternately, our model can be viewed as having random ferromagnetic interactions.

The restriction we are going to make in this paper is the following. In the Migdal-Kadanoff version, the restriction is

$$
\xi_{\mathrm{i}}=\xi_{i_{1}}
$$

i.e., all random variables whose index $\mathbf{i}$ has the same first component are equal. It is easy to give a similar formulation of this condition in the diamond version of the model; the details are left to the reader.
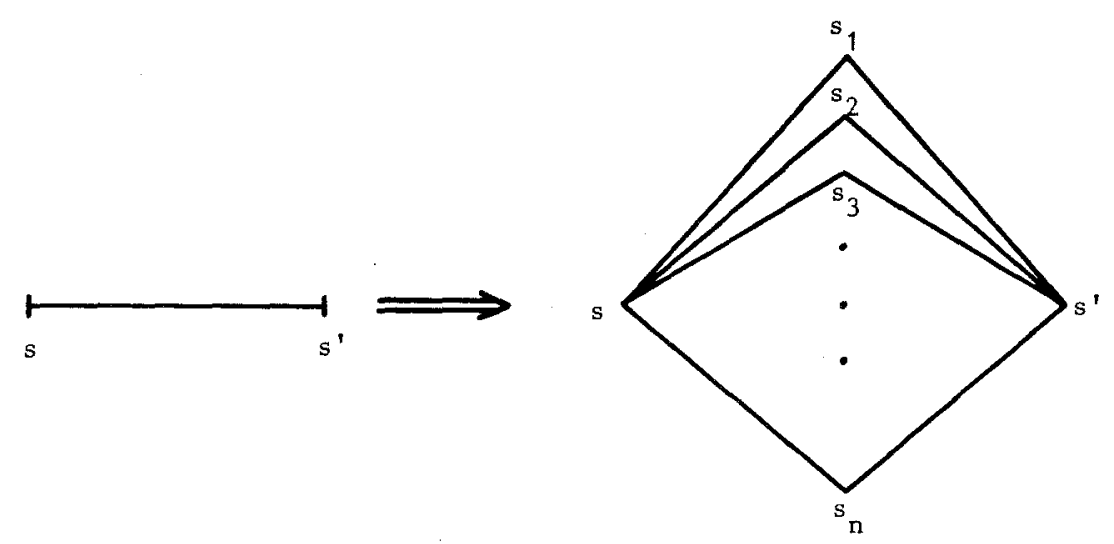

Fig. 3. Increasing the level by one. 
The point of the above models is that the Migdal renormalization transformation $^{(5)}$ is exact.

\section{RENORMALIZATION TRANSFORMATION}

In this section, we establish Migdal's recursion relations, which are exact for the models we have described. Consider $L_{N}$. The renormalization consists in summing over all spins introduced in the step leading from $L_{N-1}$ to $L_{N}$. The resulting lattice $L_{N-1}$ will have new effective (random) coupling constants. We now derive the formula for getting the new couplings $\hat{\xi}$ as a function of the old ones. It is clearly sufficient to consider the following situation (see Fig. 4).

We reemphasize that we want to sum over $s_{1}, \ldots, s_{n}$ and replace $\xi_{1}, \ldots, \xi_{n}, \xi_{1}^{\prime}, \ldots, \xi_{n}^{\prime}$ by a new random variable $\hat{\xi}$ giving an effective coupling between $s$ and $s^{\prime}$.

It is useful to introduce the random variables $x_{i}=\tanh \left(\xi_{i}\right), x_{i}^{\prime}$ $=\tanh \left(\xi_{i}^{\prime}\right)$. Moreover, we shall assume that the inverse temperature has been absorbed in the definition of the $\xi_{i}$. We then have to compute

$$
\begin{aligned}
I & =\sum_{\substack{s_{1}, \ldots, s_{n} \\
= \pm 1}} \prod_{i=1}^{n}\left(e^{\xi_{i} s_{i}} e^{\xi_{i} s_{i} s^{\prime}}\right) \\
& =\sum_{\substack{s_{1}, \ldots, s_{n} \\
= \pm 1}} \prod_{i=1}^{n} \cosh \xi_{i} \cdot \cosh \xi_{i}^{\prime} \cdot\left(1+x_{i} s s_{i}\right)\left(1+x_{i}^{\prime} s_{i} s^{\prime}\right)
\end{aligned}
$$

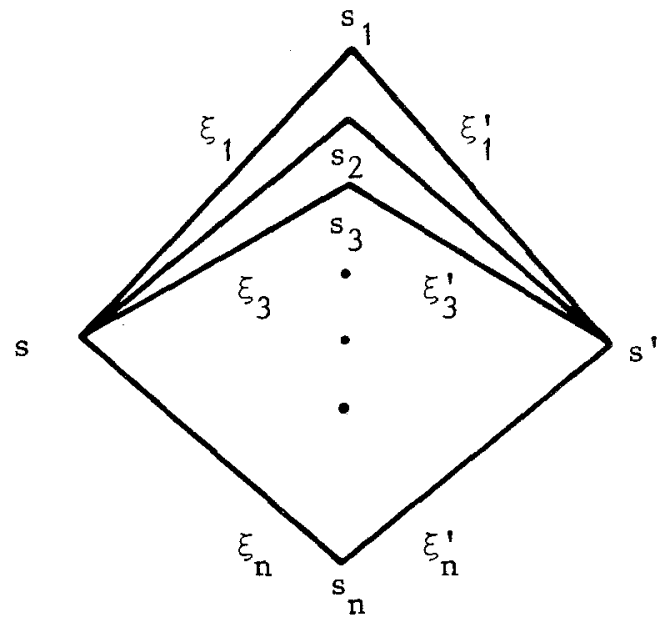

Fig. 4. Labeling one diamond. 
The factors $\cosh \xi_{i} \cdot \cosh \xi_{i}^{\prime}$ will eventually disappear in the normalization of the partition function. We shall omit them henceforth. The quantity to study is thus

$$
\begin{aligned}
& \prod_{i=1}^{n} \sum_{s_{i}= \pm 1}\left(1+x_{i} s s_{i}\right)\left(1+x_{i}^{\prime} s_{i} s^{\prime}\right) \\
&=2^{n} \prod_{i=1}^{n} \frac{1}{\cosh \left[\tanh ^{-1}\left(x_{i} x_{i}^{\prime}\right)\right]} \\
& \quad \times \prod_{i=1}^{n}\left\{\cosh \left[\tanh ^{-1}\left(x_{i} x_{i}^{\prime}\right)\right]+\sinh \left[\tanh ^{-1}\left(x_{i} x_{i}^{\prime}\right)\right] s s^{\prime}\right\} \\
&=2^{n} \prod_{i=1}^{n} \frac{1}{\cosh \left[\tanh ^{-1}\left(x_{i} x_{i}^{\prime}\right)\right]} \exp \left[\sum_{i=1}^{n} \tanh ^{-1}\left(x_{i} x_{i}^{\prime}\right) s s^{\prime}\right] \\
&= 2^{n} \cosh \left[\sum_{i=1}^{n} \tanh ^{-1}\left(x_{i} x_{i}^{\prime}\right)\right] \frac{1+\tanh \left[\sum_{i=1}^{n} \tanh ^{-1}\left(x_{i} x_{i}^{\prime}\right)\right] s s^{\prime}}{\prod_{i=1}^{n} \cosh \left[\tanh ^{-1}\left(x_{i} x_{i}^{\prime}\right)\right]}
\end{aligned}
$$

We again omit the factors which do not depend on $s, s^{\prime}$ (and which disappear in the normalization), and we get the transformation for the $\xi$ :

$$
\zeta=\sum_{i=1}^{n} \tanh ^{-1}\left[\tanh \left(\xi_{i}\right) \tanh \left(\xi_{i}^{\prime}\right)\right]
$$

In the case of interest for this paper, the $\xi_{i}$ are equal and so are the $\xi_{i}^{\prime}$ [say, to $\left.\left(\xi, \xi^{\prime}\right)\right]$ and we get

$$
\zeta=n \tanh ^{-1}\left[\tanh (\xi) \tanh \left(\xi^{\prime}\right)\right]
$$

or, in terms of the $x$,

$$
z=\tanh \left[n \operatorname{tahn}^{-1}\left(x x^{\prime}\right)\right]
$$

It is useful to denote

$$
q_{n}(s)=\tanh \left[n \tanh ^{-1}(s)\right]
$$

If all the $x_{i}$ have probability density $f$, then the probability density of the renormalized coupling $z$ will be

$$
\frac{1}{\left|q_{n}^{\prime}\left(q_{n}^{-1}(t)\right)\right|} \int_{-1}^{1} f(x) f\left(\frac{q_{n}^{-1}(t)}{x}\right) \frac{d x}{|x|}
$$

This transformation is the main object of study of our paper. 
For completeness, we also give the renormalization of a correlation function, for one spin. This quantity is given by

$$
\begin{aligned}
\mathscr{J}\left(s_{1}\right) & =\frac{1}{I} \sum_{s_{1}, \ldots, s_{n}} s_{1} \prod_{i=1}^{n} \cosh \left(\xi_{i}\right) \cosh \left(\xi_{i}^{\prime}\right)\left(1+x_{i} s s_{i}\right)\left(1+x_{i}^{\prime} s_{i} s^{\prime}\right) \\
& =\frac{x_{1}\left(1-x_{1}^{\prime 2}\right) s+x_{1}^{\prime}\left(1-x_{1}^{2}\right) s^{\prime}}{1-x_{1}^{2} x_{1}^{\prime 2}} \cdot\left\{1+\tanh \left[\sum_{i=1}^{n} \tanh ^{-1}\left(x_{i} x_{i}^{\prime}\right) s s^{\prime}\right]\right\}
\end{aligned}
$$

- The identities (3.1) and (3.2) will allow us to compute explicitly the models $L_{N}$ by $N$-fold iteration, and we shall be able to take the thermodynamic limit.

\section{BEHAVIOR UNDER RENORMALIZATION}

In this section, we investigate the action of the renormalization transformation on the probability law (i.e., the probability density).

It is easy to verify that the transformation (3.1) has three fixed probability laws, given by

$$
\begin{gathered}
\delta_{0} \\
\left(\delta_{1}+\delta_{-1}\right) / 2
\end{gathered}
$$

and

$$
\left(\delta_{a_{n}}+\delta_{-a_{n}}\right) / 2, \quad \text { where } \quad q_{n}\left(a_{n}^{2}\right)=a_{n}
$$

We do not know whether there are other fixed points, but we conjecture that there are none. We now prove some results about the basin of attraction of these three fixed points. Our analysis is not as complete as one could wish, but in the simplified case of ${ }^{(7)}$ we shall be able to get a picture which is probably the same as the one to be expected for the transformation (3.1).

We shall denote by $x_{m}$ the random coupling obtained after $m$ steps of renormalization from $x_{0}$. If $x_{m}^{\prime}$ denotes an independent identical copy of $x_{m}$, then $x_{m+1}$ is in fact nothing else than

$$
x_{m+1}=q_{n}\left(x_{m} \cdot x_{m}^{\prime}\right) \text {. }
$$

We shall now study the limiting behavior of this recursion, and for this purpose, we state some preliminary estimates. Henceforth we shall fix $n$, and denote $q=q_{n}, a=a_{n}$. We also define

$$
P_{m}(x)=\operatorname{Prob}\left\{\left|x_{m}\right| \leqslant x\right\}
$$


Lemma 4.1. The following inequalities hold:

(Ei) One has $P_{m+1}(x) \geqslant P_{m}(h(x))^{2}$, where $h(x)=\left[q^{-1}(x)\right]^{1 / 2}$.

(Eii) If $P_{m}(y)=P_{m}(a)$ for $y \geqslant a$ then $0 \leqslant x \leqslant a$, implies $P_{m+1}(x)$ $\geqslant 2 P_{m}(g(x))-P_{m}(g(x))^{2}$, where $g(x)=q^{-1}(x) / a$.

(Eiii) If $P_{m}(y)=0$ for $y<a$, then, for $a \leqslant x \leqslant 1, P_{m+1}(x) \leqslant$ $P_{m}(g(x))^{2}$.

(Eiv) If $P_{m}(y)=0$ for $y<a$, then, for $a \leqslant x \leqslant 1, P_{m+1}(x) \leqslant$ $2 P_{m}(h(x))$.

(Ev) $P_{m+1}(x) \geqslant 2 P_{m}\left(q^{-1}(x)\right)-P_{m}\left(q^{-1}(x)\right)^{2}$.

Proof. If we view $P_{m+1}(x)$ as a double integration, over $q\left(x_{m} x_{m}^{\prime}\right)$ $\leqslant x$, then the five inequalities are straightforward consequences of restrictions of these domains of integration. We visualize them graphically (see Fig. 5).

We can now use these estimates to prove convergence. We shall consider only even distributions.

Lemma 4.2. If $x_{0} \in[-a, a]$, almost surely, and $P_{0}(a-\epsilon)>0$ for some $\epsilon>0$, then, almost surely $x_{m} \rightarrow 0$ as $m \rightarrow \infty$.

Proof. We have $P_{0}(y)=P_{0}(a)$ for $y \geqslant a$ by assumption, and since $a$ is a repelling fixed point for $q\left(z^{2}\right)$, this implies $P_{m}(y)=P_{m}(a)$ for all $m$, and $y \geqslant a$. Thus (Ei), (Eii) apply.

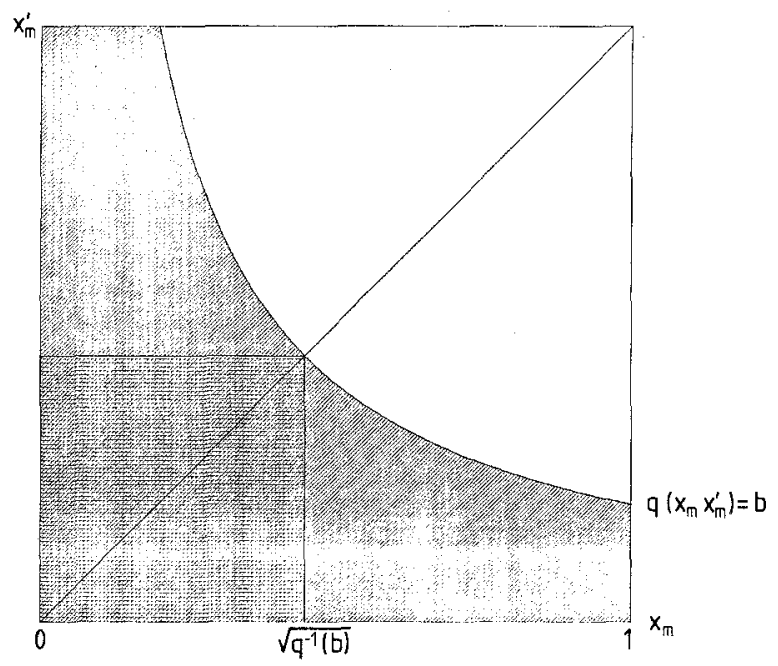

(a)

Fig. 5. Integration regions. 


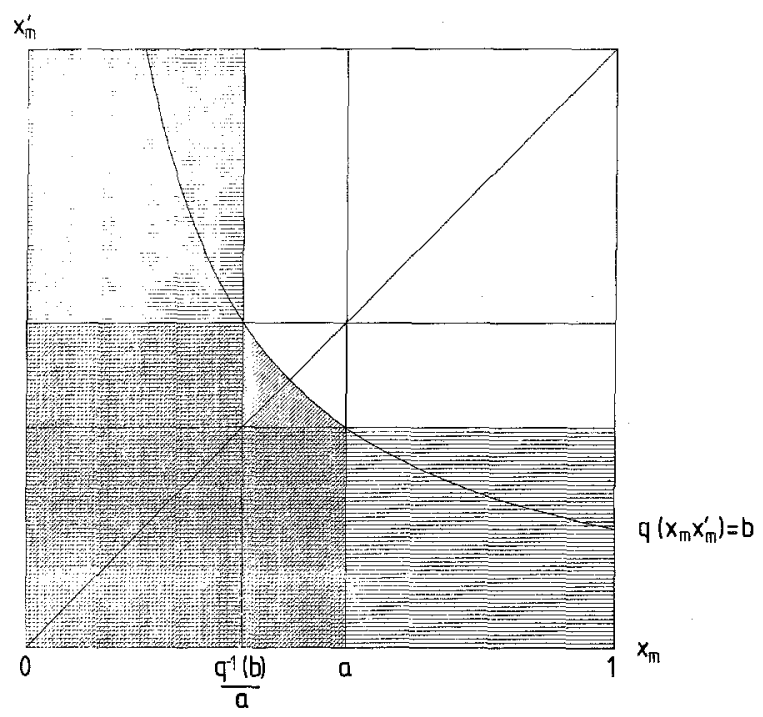

(b)

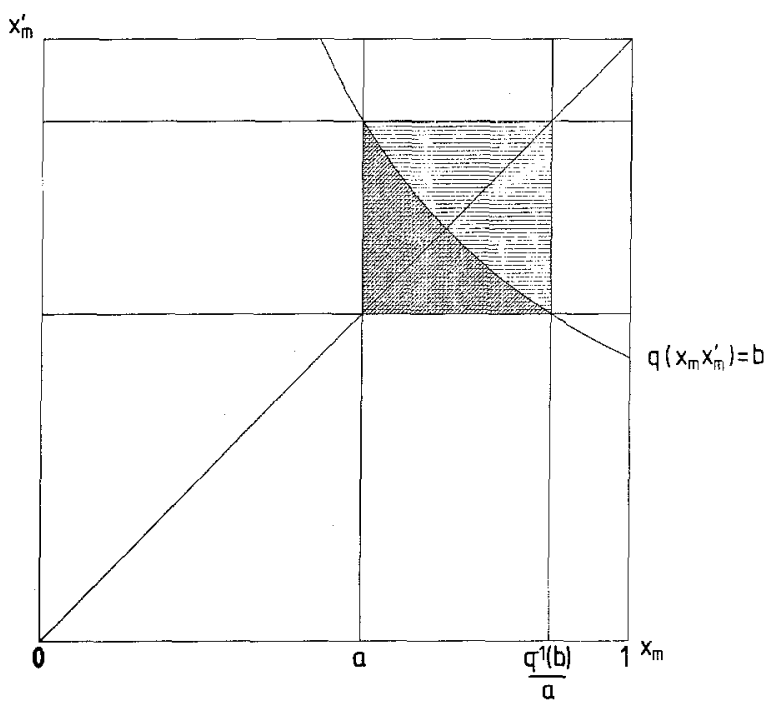

(c)

Fig. 5. Continued.

For $x \leqslant a$, we have

$$
P_{m+2}(x)=k\left(P_{m}(h \circ g(x))\right)
$$

where $k(z)=2 z^{2}-z^{4}$. The map $z \rightarrow k(z)$ has a stable fixed point at 1 , which attracts the interval $(\sigma, 1]$ where $\sigma=(\sqrt{5}-1) / 2$. Note also that $g^{-1} \circ h^{-1}$ has a stable fixed point at 0 which attracts $[0, a)$. Therefore, for 


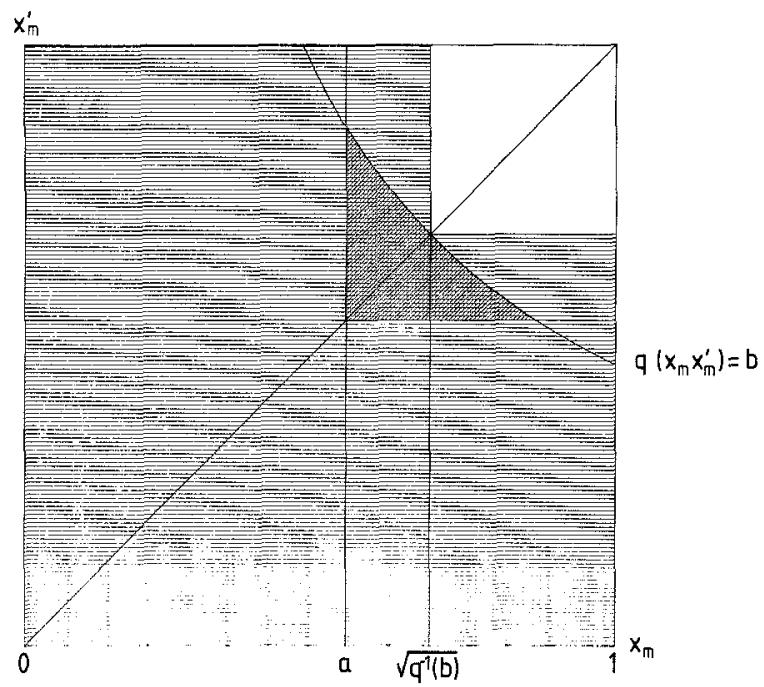

(d)

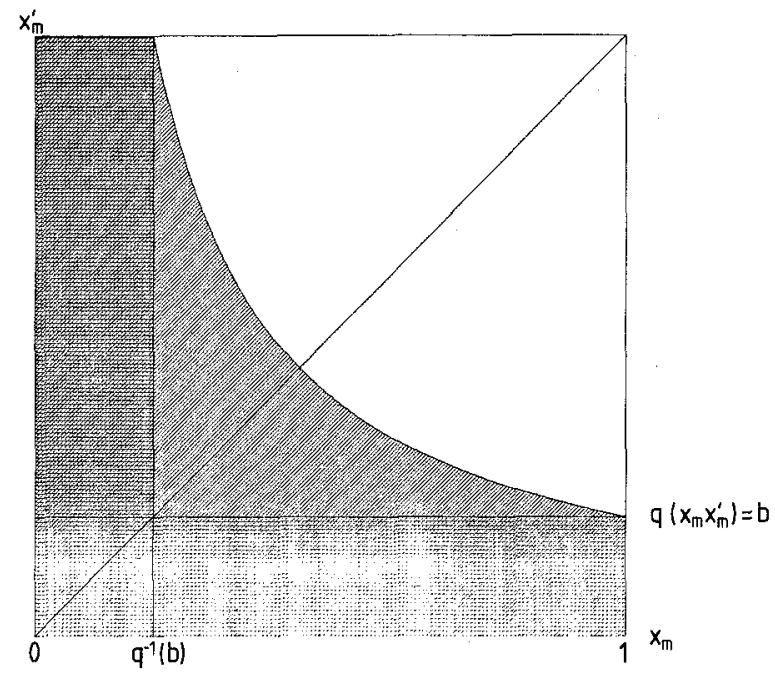

(e)

Fig. 5. Continued.

$x \in[0, a)$ we find

$$
\begin{aligned}
P_{m+2 p}(x) & \geqslant P_{m+2 p}\left(g^{-1} \circ h^{-1}(x)\right) \geqslant k\left(P_{m+2(p-1)}(x)\right) \\
& \geqslant k^{p}\left(P_{m}(x)\right)
\end{aligned}
$$

Thus if $P_{m}(x)>\sigma$, then $P_{m+2 p}(x) \rightarrow 1$ as $p \rightarrow \infty$. 
Consider now $l(x)=2 x-x^{2}$. The map $z \rightarrow l(z)$ has a stable fixed point at 1 , which attracts $(0,1]$. Since $P_{0}(a) \neq 1$, and $\left|x_{0}\right| \leqslant a$ almost surely there is a $y<a$ for which $P_{m}(y) \neq 0$. Therefore, there is an $s$ for which $l^{s}(y)>\sigma$, and hence, by (Eii), for $x=g^{-s}(y)$,

$$
P_{s}(x) \geqslant l^{s}\left(P_{0}(y)\right)>\sigma
$$

and hence,

$$
P_{s+2 p}(x) \rightarrow 1
$$

Since $P_{m}$ is monotone, and $g(x)<x$ when $x<a$, we have

$$
P_{s+2 p}\left(g^{-1}(x)\right) \rightarrow 1
$$

But this implies, by (Eii),

$$
P_{s+2 p-1}(x)^{2} \rightarrow 1
$$

and the lemma follows.

It is interesting to note that $P_{p}(y)$ in general will not converge monotonously to 1 , and in fact the nature of the convergence will depend very much on the density of $x_{0}$. We are confronted with two opposing tendencies: Lemma 4.1 tells us that the weight of the density moves toward 0 , but it also gets smaller. All these facts will become more transparent in the simplified model of Ref. 7. They are responsible for the absence of critical indices at the critical surface. The following lemma is a sort of converse of Lemma 4.2.

Lemma 4.3. Assume $\left|x_{0}\right| \geqslant a$ almost surely and $P_{0}(a) \neq 1$. Then $\left|x_{m}\right| \rightarrow 1$ almost surely, as $m \rightarrow \infty$.

Proof. The construction we give now has to go somewhat backwards. Let $\alpha=P_{0}(a)$. By assumption, we have $\alpha<1$. We denote $\alpha^{\prime}=(1+$ $\alpha) / 2(<1)$. Define $s$ by

$$
\left(\alpha^{\prime}\right)^{2^{s}}<2^{-(r+1)}
$$

Choose $b>a$ such that (i) $q^{-1}(b)<a$, (ii) $P_{0}\left(g^{s}(b)\right) \leqslant \alpha^{\prime}$, (iii) $g^{s}(b)<c$, where $c$ is the boundary of the basin of attraction of $h^{r} \circ g$. Such a $b$ exists (it suffices to choose it sufficiently close to $a$ ).

Assume now $d \in[a, 1)$, and choose $q$ such that $h^{q}(d) \leqslant b$. Then we find, using (Eiv),

$$
P_{q+m}(d) \leqslant 2^{q} P_{m}\left(h^{q}(d)\right) \leqslant 2^{q} P_{m}(b)
$$

It suffices therefore to prove that $P_{m}(b) \rightarrow 0$ as $m \rightarrow \infty$. By (ii) above

$$
P_{s}(b) \leqslant P_{0}\left(g^{s}(b)\right)^{2^{s}} \leqslant \alpha^{2^{s}}<2^{-(r+1)}
$$


Applying alternately the two inequalities (Eiii) and (Eiv) we see that

$$
\begin{aligned}
P_{s+p(r+1)}(b) & \leqslant P_{p(r+1)}\left(g^{s}(b)\right)^{2^{s}} \\
& \leqslant 2^{r} P_{(p-1)(r+1)}\left(h^{r} \circ g\right)\left(\left(g^{s}(b)\right)\right)^{2^{s} \cdot 2} \\
& \leqslant 2^{r} P_{(p-1)(r+1)}\left(g^{s}(b)\right)^{2^{s} \cdot 2}
\end{aligned}
$$

since (iii) implies $\left(h^{r} \circ g\right)\left(g^{s}(b)\right)<g^{s}(b)$. Iterating, we get

$$
P_{s+p(r+1)}(b) \leqslant 2^{r\left(2^{p}-1\right)}\left(P_{0}\left(g^{s}(b)\right)^{2^{s}}\right)^{2^{p}} \leqslant 2^{-2^{p}} \rightarrow 0
$$

by (Eii) and (Eiii). Since $h(x)<x$, for $x>a$, and $P_{m}$ is monotone, we have

$$
\begin{aligned}
P_{s+p(r+1)+q}(b) & \leqslant P_{s+p(r+1)+q}\left(h^{-(r-q+1)}(b)\right) \\
& \leqslant 2^{q} P_{m+p(r+1)}(b) \rightarrow 0, \quad \text { for } q=0, \ldots, r
\end{aligned}
$$

This completes the proof.

We can now analyze easily the temperature dependence for a subclass of random variables $\xi_{0}$ and show that there is a phase transition, as far as the random couplings are concerned. We postpone to the next section the physical aspects of this transition.

Theorem 4.4. Let $\xi_{0}$ be a random variable such that for some $\gamma_{1}, \gamma_{2}$,

$$
0<\gamma_{1} \leqslant\left|\xi_{0}\right| \leqslant \gamma_{2}<1, \quad \text { almost surely (a.s.) }
$$

If the temperature $T$ is sufficiently large, the associated sequence $x_{m}$ tends to zero, almost surely, while for $T$ sufficiently small, $\left|x_{m}\right|$ tends to 1 almost surely.

Proof. By construction, we have $x_{0}=\tanh \left(\beta \xi_{0}\right)$ with $\beta=1 / T$, and therefore

$$
\tanh \left(\beta \gamma_{1}\right) \leqslant\left|x_{0}\right| \leqslant \tanh \left(\beta \gamma_{2}\right), \quad \text { a.s. }
$$

Therefore, by Lemma 4.2 , if $\beta<\gamma_{2}^{-1} \cdot \tanh ^{-1}(a)$, we have $x_{m} \rightarrow 0$ a.s., while, by Lemma 4.3 , if $\beta>\gamma_{1}^{-1} \tanh ^{-1}(a)$, then $\left|x_{m}\right| \rightarrow 1$, a.s.

When $\xi_{0}$ is of the type described in Theorem 4.4, one could be tempted to identify the critical temperature as the largest temperature for which $\left|\tanh \left(\beta \xi_{0}\right)\right| \leqslant a$, a.s. This is, however, not the case, as can be shown by an explicit example, which we do not present here. [If $\left|\xi_{0}\right|$ is a.s. constant, the critical temperature $i s$ characterized by $\left|\tanh \left(\beta \xi_{0}\right)\right|=a$, a.s.]

We next discuss a more detailed issue-a description of the basin of attraction of $x=0$. Our description is not complete, but it shows that the domain of attraction has a structure which is not very simple in the $L_{1}$ topology. This is due to the nondifferentiability of the renormalization map in this space. 
We present here the result only for the case $n=2$, i.e., $q=q_{2}$. Other cases are similar, and better constants can be read off the proof.

Lemma 4.5. If, for some $0<\alpha \leqslant 1 / 128$, one has $P_{0}(\alpha)>4 \alpha$, then $x_{m} \rightarrow 0$ almost surely.

Proof. By the inequalities (Ei), (Ev), we have

$$
P_{p+1+m}(x) \geqslant v \circ u^{p}\left(P_{m}\left(q^{-p} \circ h(x)\right)\right)
$$

where $v(z)=z^{2}, u(z)=2 z-z^{2}$. Below, we shall show that

$$
v \circ u^{p} \text { attracts }\left(\tau_{p} / 4^{p}, 1\right] \text { to } 1
$$

and $\tau_{p} \rightarrow 1$ as $p \rightarrow \infty\left(\tau_{3} \sim 1.45\right)$.

On the other hand, since $q(x)=q_{2}(x)=2 x /\left(1+x^{2}\right)$, we have $q^{-1}(x)$ $>x / 2$ and $h(x)>(x / 2)^{1 / 2}$. Therefore, if $x=1 / 2^{2 p+1}$, then $q^{-p} \circ h(x)$ $>x$. Assume now

$$
P_{0}\left(1 / 2^{2 p+1}\right)>2 \tau_{p} / 4^{p}
$$

Then, setting $x=1 / 2^{2 p+1}$, we have

$$
\begin{aligned}
P_{p s}(x) & \geqslant v \circ u^{p}\left(P_{p(s-1)}\left(q^{-p} \circ h(x)\right)\right) \\
& \geqslant v \circ u^{p}\left(P_{p(s-1)}(x)\right) \geqslant\left(v \circ u^{p}\right)^{s}\left(2 \tau_{p} / 4^{p}\right) \rightarrow 1
\end{aligned}
$$

This implies by (Ei),

$$
P_{p s+1}\left(q\left(w^{2}\right)\right) \geqslant P_{p s}(w) \rightarrow 1
$$

But $q\left(1 / 2^{2 p+1}\right)^{2}<2\left(2^{2 p+1}\right)^{2}$, and repeating this argument, we see that $P_{m}(x) \rightarrow 1$ for all $x>0$. It remains to prove (4.1). This is an easy consequence of the inequality, obtained by induction,

$$
u^{p}(z) \geqslant 2^{p} z-2^{p-1}\left(2^{p}-1\right) z^{2}
$$

and of

$$
v \circ u^{p}(z) \geqslant 4^{p} z^{2}-4^{p}\left(2^{p}-1\right) z^{3}
$$

\section{SPIN OBSERVABLES}

In this section, we investigate the behavior of the expectation of the spin when the "volume" of the lattice tends to infinity. We fix the values of the spin at the two extreme points of the lattice, thereby choosing the boundary conditions. If $s$ is a spin which is not one of the above two, we shall denote by $\langle s\rangle$ its expectation for fixed boundary condition, and fixed values of the random couplings. We show that the expectation $E\left(\langle s\rangle^{2}\right)$ (i.e., 
average over couplings) of $\langle s\rangle^{2}$ satisfies

$$
E\left(\langle s\rangle^{2}\right) \begin{cases}=0, & \text { at high temperature } \\ \neq 0, & \text { at low temperature }\end{cases}
$$

and

$$
E(\langle s\rangle)=0 \quad \text { at all temperatures }
$$

Note that the above argument shows a transition for the EdwardsAnderson $^{(1,3)}$ parameter. We shall also see that the value of the EdwardsAnderson parameter is independent of the boundary conditions.

Consider the lattice $L_{N}$ and a fixed spin variable $s_{0}$ which has been "created" at level $N$. The variable $s_{0}$ has two neighboring sites, exactly one of which has been created at level $N-1$. We call it $s_{1}$ and we call the other neighbor $s_{1}^{\prime}$. Considering now $s_{1}$ as a fixed spin in $L_{N-1}$, we find its neighbors $s_{2}$ and $s_{2}^{\prime}$ in the same fashion as before, and continuing inductively, we find two chains $s_{0}, s_{1}, \ldots, s_{N}$ and $s_{0}, s_{1}^{\prime}, s_{2}^{\prime}, \ldots, s_{N}^{\prime}$ of spins. Note that all $s_{i}$ are distinct, but some of the $s_{i}^{\prime}$ may coincide. (See Fig. 6.) Note also that either $s_{m+1}=s_{m}^{\prime}$ or $s_{m+1}^{\prime}=s_{m}^{\prime}$.

To these chains of spins, we associate functions of the form

$$
F_{i}\left(s_{i}, s_{i}^{\prime}\right)=a_{i} s_{i}+b_{i} s_{i}^{\prime}
$$

where the $a_{i}, b_{i}$ are real functions depending on the couplings $x_{0}$. We are interested in $\left\langle F_{i}\left(s_{i}, s_{i}^{\prime}\right)\right\rangle$ [i.e., the canonical expectation of the observable $\left.F_{i}\left(s_{i}, s_{i}^{\prime}\right)\right]$. We have the following important identity:

$$
\left\langle A s_{m}+B s_{m}^{\prime}\right\rangle=\left\langle A^{\prime} s_{m+1}+B^{\prime} s_{m+1}^{\prime}\right\rangle
$$

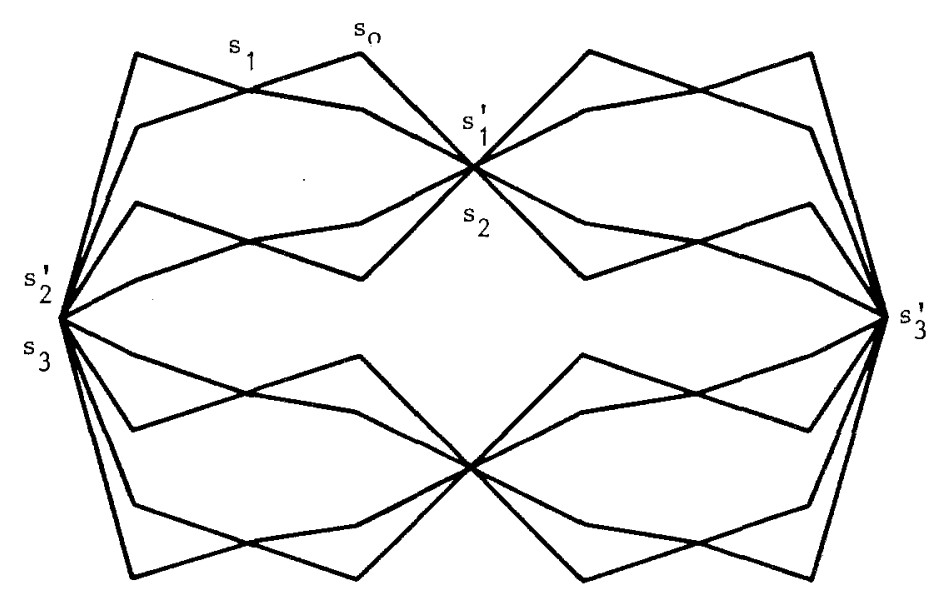

Fig. 6. Labeling of the spins. 
where

$$
\begin{aligned}
& A^{\prime}=B+A \frac{x_{m}\left(1-x_{m}^{\prime 2}\right)}{1-x_{m}^{2} x_{m}^{\prime 2}} \\
& B^{\prime}=A \frac{x_{m}^{\prime}\left(1-x_{m}^{2}\right)}{1-x_{m}^{2} x_{m}^{\prime 2}}
\end{aligned}
$$

and

$$
\begin{aligned}
& A^{\prime}=A \frac{x_{m}\left(1-x_{m}^{\prime 2}\right)}{1-x_{m}^{2} x_{m}^{\prime 2}} \\
& B^{\prime}=B+A \frac{x_{m}^{\prime}\left(1-x_{m}^{2}\right)}{1-x_{m}^{2} x_{m}^{\prime 2}} \quad \text { if } \quad s_{m+1}^{\prime}=s_{m}^{\prime}
\end{aligned}
$$

Here, $x_{m}$ and $x_{m}^{\prime}$ are two identical, independent random couplings obtained from $x_{0}$ through $m$-fold application of the RG transformation, $x_{m}=$ $\tanh \left(T^{m} \xi\right)$. The above identities are immediately obtained by induction when summing over all spins at level $N-m$.

Namely,

$$
\begin{aligned}
\left(1+x_{m} s_{m} s_{m+1}\right)\left(1+x_{m}^{\prime} s_{m} s_{m+1}^{\prime}\right)= & \left(1+x_{m} x_{m}^{\prime} s_{m+1} s_{m+1}^{\prime}\right) \\
& \times\left(1+\frac{x_{m} s_{m+1}+x_{m}^{\prime} s_{m+1}^{\prime}}{1+x_{m} x_{m}^{\prime} s_{m+1} s_{m+1}^{\prime}} s_{m}\right)
\end{aligned}
$$

and, summing over $s_{m}$ (and taking the mean, we get)

$$
\begin{gathered}
\sum_{s_{m}}\left(1+\frac{x_{m} s_{m+1}+x_{m}^{\prime} s_{m+1}^{\prime}}{1+x_{m} x_{m}^{\prime} s_{m+1} s_{m+1}^{\prime}} s_{m}\right) \cdot\left(A s_{m}+B s_{m}^{\prime}\right) \\
=A\left(\frac{x_{m} s_{m+1}+x_{m}^{\prime} s_{m+1}^{\prime}}{1+x_{m} x_{m}^{\prime} s_{m+1} s_{m+1}^{\prime}}\right)+B s_{m}^{\prime}
\end{gathered}
$$

The equations follow from $s_{m}^{\prime}=s_{m+1}$ (resp. $=s_{m+1}^{\prime}$ ).

Given $s_{0}$ and $A_{0} \neq 0$, we therefore find, when $B_{0}=0$,

$$
\begin{aligned}
\left\langle A_{0} s_{0}\right\rangle & =\left\langle A_{1} s_{1}+B_{1} s_{1}^{\prime}\right\rangle \\
& =\cdots \\
& =A_{n} s_{N}+B_{N} s_{N}^{\prime}
\end{aligned}
$$

where the $A_{i}, B_{i}$ are recursively obtained by the above relations, and are random variables in all $x_{j}, x_{j}^{\prime}=\tanh \left(\xi_{j}\right), \tanh \left(\xi_{j}^{\prime}\right)$ created above level $i$.

Lemma 5.1. $E\left(A_{m} B_{m}\right)=E\left(A_{m}\right)=E\left(B_{m}\right)=0$. 
Proof. We shall show below, recursively, that

$$
E\left(A_{m} x_{m} f\left(x_{m}^{2}\right)\right)=E\left(B_{m} x_{m} f\left(x_{m}^{2}\right)\right)=0
$$

for any function $f$.

Using this result, and the induction rule for forming $A_{m+1}$ from $A_{m}, B_{m}$, we get, in case $s_{m+1}=s_{m}^{\prime}$

$$
E\left(A_{m+1}\right)=E\left(A_{m}\right)+E\left(B_{m} x_{m} \frac{1-x_{m}^{2}}{1-x_{m}^{2} x_{m}^{\prime 2}}\right)
$$

from which the assertion $E\left(A_{m+1}\right)=0$ follows. Similarly, $E\left(B_{m+1}\right)=0$. Finally,

$$
\begin{aligned}
E\left(A_{m+1} B_{m+1}\right)= & E\left(A_{m} B_{m} x_{m}^{\prime} \frac{1-x_{m}^{2}}{1-x_{m}^{2} x_{m}^{\prime 2}}\right) \\
& +E\left(B_{m}^{2}\left[\frac{x_{m} x_{m}^{\prime}\left(1-x_{m}^{2}\right)\left(1-x_{m}^{\prime 2}\right)}{\left(1-x_{m}^{\prime 2} x_{m}^{2}\right)^{2}}\right]\right)=0
\end{aligned}
$$

by (5.1). It remains to prove (5.4). But this is obvious since

$$
E\left(A_{m} x_{m} f\left(x_{m}^{2}\right)\right)=E\left(A_{m-1} x_{m-1} \Phi\left(x_{m-1}^{2}, x_{m-1}^{\prime 2}\right)\right)
$$

where

$$
\Phi\left(x_{m-1}^{2}, x_{m-1}^{\prime 2}\right)=x_{m-1}^{2}\left(1-x_{m-1}^{\prime 2}\right) f\left(q_{n}\left(x_{m-1} x_{m-1}^{\prime}\right)^{2}\right)
$$

and so the result follows by induction.

We next show recursively that

$$
A_{m+1} B_{m+1} x_{m+1} \geqslant 0 \quad \text { a.s. }
$$

Indeed, this follows again by induction, if we use

$$
\begin{aligned}
A_{m+1} B_{m+1} x_{m+1}= & \frac{x_{m} A_{m} B_{m} x_{m}^{\prime}}{1-x_{m}^{2} x_{m}^{\prime 2}} \frac{\left(1-x_{m}^{2}\right) x_{m}}{x_{m}^{2}} q_{n}\left(x_{m} x_{m}^{\prime}\right) \\
& +B_{m}^{2} \frac{\left(1-x_{m}^{2}\right)\left(1-x_{m}^{\prime 2}\right)}{x_{m}^{2}} q_{n}\left(x_{m} x_{m}^{\prime}\right) \\
\geqslant & 0
\end{aligned}
$$

since $x_{m} x_{m}^{\prime} q_{n}\left(x_{m} x_{m}^{\prime}\right) \geqslant 0$ and $A_{m} B_{m} x_{m} \geqslant 0$, a.s.

Therefore,

$$
\left|A_{m+1}\right|+\left|B_{m+1}\right|=\left|A_{m}\right|+\left|B_{m}\right| \frac{\left|x_{m}\right|+\left|x_{m}^{\prime}\right|}{1+\left|x_{m} x_{m}^{\prime}\right|}
$$

as is easily seen. 
Assume now $\left|x_{m}\right| \rightarrow 1$, exponentially fast (this certainly happens for the $x_{0}$ of Theorem 4.4).

Then

$$
\left|A_{m}\right|+\left|B_{m}\right| \geqslant\left(\left|A_{0}\right|+\left|B_{0}\right|\right) \text { const, }
$$

by (5.5) and this (positive) constant does not depend on $m$. If we set $A_{0}=1$, $B_{0}=0$, and we now take $m=N$, we obtain

$$
\left\langle s_{0}\right\rangle=\left\langle A_{N} s_{N}+B_{N} s_{N}^{\prime}\right\rangle=A_{N} s_{N}+B_{N} s_{N}^{\prime}
$$

From Lemma 5.1 this implies $E\left(\left\langle s_{0}\right\rangle\right)=0$. On the other hand,

$$
\begin{aligned}
E\left(\left\langle s_{0}\right\rangle^{2}\right) & =E\left(A_{n}^{2}+B_{n}^{2}\right)+E\left(A_{N} B_{N}\right) s_{N} s_{N}^{\prime} \\
& =E\left(A_{n}^{2}+B_{N}^{2}\right) \geqslant \frac{1}{2} E\left(\left(\left|A_{N}\right|+\left|B_{N}\right|\right)^{2}\right)>0
\end{aligned}
$$

Hence we have shown for any fixed $s_{N}, s_{N}^{\prime}$ the following:

Theorem 5.2. If $\left|x_{m}\right| \rightarrow 1$ or 0 a.s. exponentially fast then

$$
E(\langle s\rangle)=0
$$

$E\left(\langle s\rangle^{2}\right)$ has a limit which is not zero if $\left|x_{m}\right| \rightarrow 1$ and zero otherwise.

\section{ACKNOWLEDGMENTS}

P.C. and J.-P.E. would like to thank H. Weinberger and G. Sell for their kind hospitality at IMA where this work was begun. J.-P.E. wishes to thank the IHES for its warm hospitality. P.C. acknowledges partial support from the Fonds National Suisse.

\section{REFERENCES}

1. G. Toulouse, Frustrations et désordres: problèmes nouveaux en mécanique statistique. Histoire des verres de spin. Congrès de la Société Française de Physique, Clermont-Ferrand 1981 (Les Editions de Physique 1982).

2. R. B. Griffiths and M. Kaufman, Spin systems on hierarchical lattices, Phys. Rev. B 26:5022-5032 (1982).

3. A. C. Van Enter and R. B. Griffiths, The order parameter in a spin glass, Commun. Maih. Phys. 90:319 (1983).

4. L. P. Kadanoff, Notes on Migdal's recursion formulas, Ann Phys. (N.Y.) 100:359-394 (1976).

5. A. D. Migdal, Phase transitions in gauge and spin lattice systems, Zh. Eksp. Teor. Fiz. 69:1457-1465 (1975).

6. D. Sherrington and S. Kirkpatrick, Phys. Rev. Lett. 35:1792 (1975).

7. P. Collet, J-P. Eckmann, V. Glaser, and A. Martin, Commun. Math. Phys. (in print).

8. P. Collet and J.-P. Eckmann, A spin-glass model with random couplings, Commun. Math. Phys. 93:379-406 (1984). 